\title{
The Use of Facial CT for the Evaluation of a Suspected Simple Dentoalveolar Abscess in the Emergency Department
}

\author{
Waqas Shuaib, MD; Mariyam Hashmi, MD; Arvind Vijayasarathi, MD, MPH, MBA; \\ Jay Arunkumar, DMD; Sabeen Tiwana, DMD; and Faisal Khosa, MD
}

\begin{abstract}
Introduction: Facial computed tomography $(\mathrm{CT})$ is often performed in the emergency department as a part of the diagnostic workup for patients presenting with the signs and symptoms of dentoalveolar abscess (DA). This investigation evaluated the diagnostic yield of the facial CT and its effects on management change in patients suspected of DA. Furthermore, we assessed secondary consequences of routine facial CT use in this population by using turn-around time (TAT), cost, and radiation exposure as the key parameters.
\end{abstract}

Material and Methods: We retrospectively reviewed records of suspected DA patients over a 5-year period, from June 2008 to June 2013. TAT was calculated for patients from the time the examination was ordered by the emergency department physician, to the time the report was finalized by the attending radiologist. Effective radiation dose for facial CT was calculated by multiplying dose length product by the standard conversion coefficient $\mathrm{K}(\mathrm{K}=0.002 \mathrm{I} \mathrm{mv} / \mathrm{mGy} \times \mathrm{cm})$. CT cost was included for reference but was not analyzed extensively due to the lack of standardization of costs across the various institutions.

Results: Our investigation consisted of II7 patients; 75 males of average age $4 \mathrm{I}( \pm \mathrm{I} 2)$ years and 42 females of average age $44( \pm 17)$ years. Out of the total 117 patients that underwent CT for the suspicion of a simple DA, only a single individual (0.85\%) underwent a management change. Mean TAT of facial CT was II0 ( \pm 63$)$ minutes, and a median TAT was $87( \pm 7 \mathrm{I})$ minutes. Average effective radiation dose for a facial CT was $2.4( \pm 0.4) \mathrm{mSv}$. Approximate estimated cost of a contrast enhanced facial CT was $\$ 253$ and non-contrast facial CT was $\$ 209$.

Conclusion: Our study shows that routine use of facial CT has a very limited diagnostic value in the workup of simple DA and rarely results in management change. The overall impact on patient management is miniscule in the context of prolonged TAT, radiation exposure, and adding to the rising medical costs.

Keywords: Facial CT; Turnaround time; Radiation; Cost-analysis

dontogenic infections leading to a simple dentoalveolar abscess (DA) are a common, non-traumatic dental condition (NTDC) encountered in emergency departments (ED). Simple DA is classified into two main types; the endodontic (periapical) abscess and the periodontal abscess. Endodontic abscess forms secondary to dental caries, after the necrosis of the dental pulp and subsequent infection of the root canal. In a periodontal abscess, the infection originates from the periodontal tissues or the gingiva. ${ }^{1}$ The ED sometimes serves as the primary care setting for the workup of simple DA, instead of outpatient dental clinics. This trend has resulted in an increase in the dental related ED visits, as a percentage of all ED visits. ${ }^{1}$
Corresponding Author: Waqas Shuaib, MD; Department of Radiology and Imaging Sciences; 550 Peachtree Street NE; Atlanta, GA 30308; Tel: (404) 686-5957; Fax: (404) 686-4498; Email:waqas.shuaib@emory.edu
Received: May 3, 2014

Revised: July 14, 2014

Accepted: July 24,2014
Disclosure: Dr. Khosa is the

American Roentgen Ray

Society Scholar (2013-20I5) 
Published literature has advocated the use of the simple, cheap, and familiar technique of panoramic radiographs as the standard radiological technique in evaluating simple DA. ${ }^{2}$ However, in most ED settings, facial computed tomography (CT) has superseded the use of panoramic radiographs, because of its availability, easy access, and potential to offer greater anatomic detail. There are, however, issues associated with the routine use of $\mathrm{CT}$ in terms of increased turnaround time (TAT), radiation exposure, and technical/professional fees associated with routinely utilizing facial $\mathrm{CT}$ in the workup of DA. Okunseri et $\mathrm{al}^{3}$ state that patients who present to the ED with an NTDC would be better managed in an outpatient dental office setting, given the availability of definitive care and the likelihood of continuity of patient care. While there is extensive variability in the management and diagnostic algorithms applied to suspected simple DA in the ED setting across the country, advanced imaging has become much more common in the age of widely available multidetector CTs. Inappropriate and continuous use of the ED for NTDC burdens the health care system by lending to overcrowding, increasing care cost, longer wait times for patients with other urgent health conditions, and increased exposure to ionizing radiation. ${ }^{1,3}$

Though outpatient dental treatment would be ideal, in the event that these patients present to the ED, rapid, efficient, and effective care delivery is of the utmost importance. Our investigation examined the diagnostic utility of facial $\mathrm{CT}$ in patients with suspected simple DA, as well as the TAT, radiation exposure, and associated financial costs. The central hypothesis of this investigation was that facial CT rarely results in a change of patient management in the clinical setting of suspected simple DA.

\section{Materials and Methods}

This retrospective investigation was conducted with institutional review board approval and was compliant with HIPAA. The requirement for written informed consent was waived because of the retrospective nature of the investigation.

\section{Study Population}

We conducted our investigation at an urban universityaffiliated teaching hospital. We queried the healthcare enterprise data warehouse and the hospital information system for CT examinations performed in the ED with the diagnostic fields of dental abscess or dental infection. A thorough ED chart review was performed to determine the subset of patients that were suspected of having a simple DA based on their clinical signs and symptoms. These patients commonly presented with one or more of following: pyrexia, tooth pain, facial swelling, dysphagia, trismus, dyspnea, or tender cervical lymphadenopathy. All patients who presented to the hospital during a consecutive 5-year period (from June 2008 to June 2013) with the previously mentioned signs and symptoms were included. Patients who were under the age of 18 , or those who presented with acute respiratory distress, history of head and neck trauma or malignancy, or required immediate surgical intervention due to suspected complicated DA were excluded.

\section{Study Protocol}

A board-certified radiologist with fellowship training in neuroradiology reviewed the original radiographic reports to determine the definitive diagnosis concluded by the imaging studies. A facial CT was coded abnormal if the initial interpreting radiologist described the findings of apical periodontitis, submandibular or sublingual abscess, radicular cyst formation, periodontal ligament destruction, or bone erosion. Our research staff reviewed surgical notes, patient discharge summaries, and ED visit notes to determine whether there was a change in management based on the use of facial CT. A change in management was defined as a hospital admission for any surgical procedure including abscess drainage immediately following the facial CT interpretation. Generally, routine uncomplicated DA is managed in the ED by prescribing antibiotics and analgesics, and patients are discharged with a dental office referral.

\section{Time Comparison}

For patients who underwent facial CT, TAT was calculated as the number of minutes from the time the study was ordered to the time at which the report was finalized by an attending radiologist. This information was captured directly from the electronic data warehouse, as every order is time-stamped, and radiology examinations are time-stamped at every step (started, completed, dictated, and finalized).

\section{Estimation of Radiation Dose}

The CT scanner recorded dose length product (DLP), which was used to calculate the effective radiation dose. Effective radiation dose $(\mathrm{mSv})$ was estimated by multiplying DLP collected from the CT scanner with the standard conversion coefficient $(\mathrm{K})(\mathrm{K}=0.0021 \mathrm{mSv} / \mathrm{mGy} \times \mathrm{cm}){ }^{4,5}$ The DLP varied amongst our population based on the number of slices necessary to include the region of interest in different sized patients.

\section{Imaging Cost}

True cost of an imaging study is very difficult to ascertain and can vary widely across, and even sometimes within, institutions based on a variety of factors. On a national scale, global facility charges (technical and professional components) for specific imaging studies vary based on frequently negotiated agreements between hospitals and payers. As such, we chose to use national average Medicare Part B Physician Fee Schedule payments for professional and technical component services as a surrogate of the cost for both CT maxillofacial without contrast and CT maxillofacial with contrast (CPT codes 70486 and 70487, respectively).

\section{Statistical Analysis}

The analysis was performed using standard software (SAS, version 9.3; SAS, Cary, NC). Continuous variables were expressed as mean (standard deviation). Categorical variables were expressed as frequency, $\mathrm{n}(\%)$. 


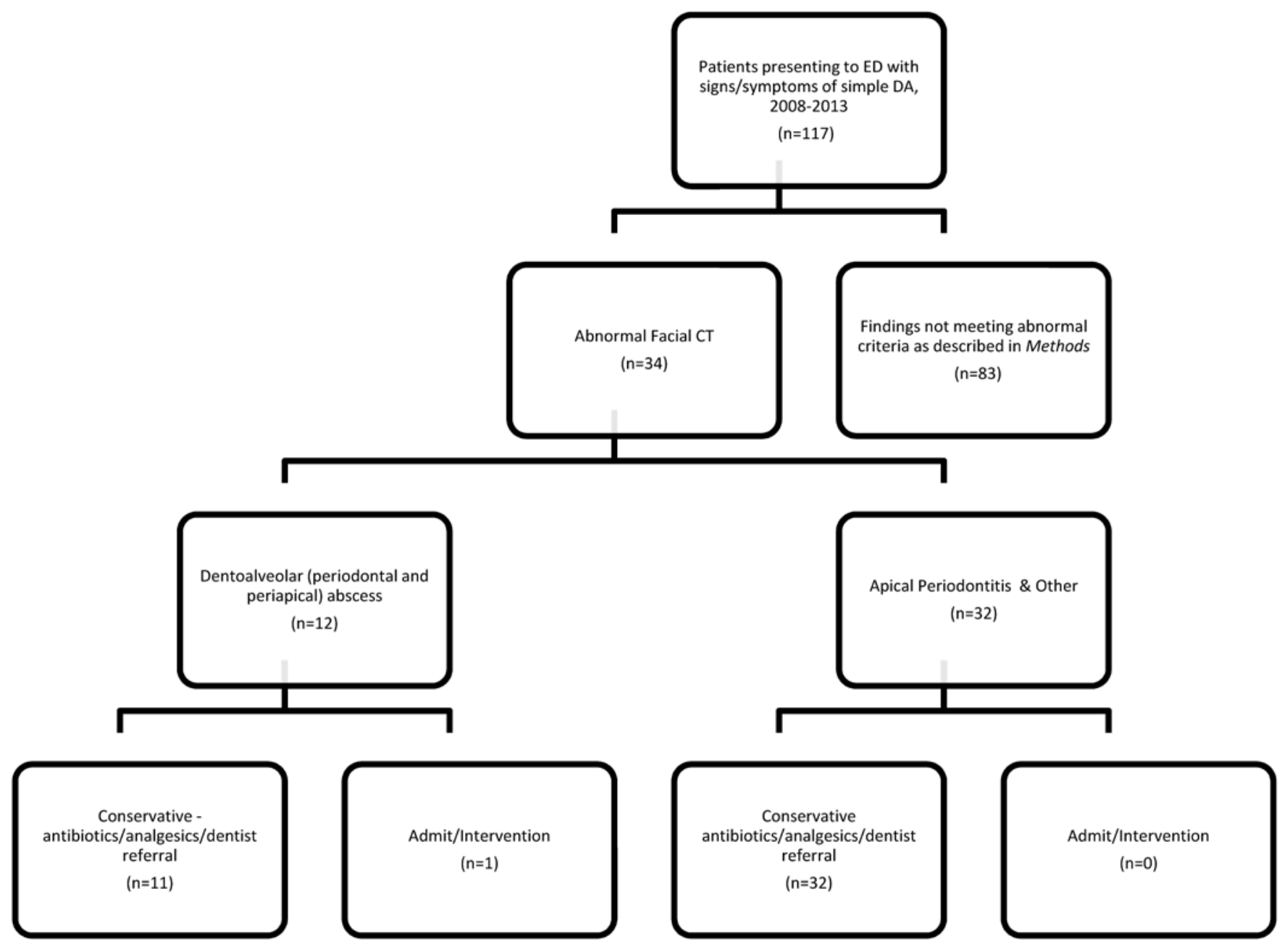

Figure 1: Facial computed tomography (CT) outcomes.

\section{Results}

During the investigation, 117 patients (75 male [64\%] and 42 female [36\%]), met the inclusion criteria described in detail in the Methods section. The average age for male patients was $41( \pm 12)$ years and for female patients was $44( \pm 17)$ years. The most common presentation related to suspect DA was toothache $(66 \%)$, followed by pyrexia $(15 \%)$, dysphagia $(9 \%)$, facial swelling $(6 \%)$, trismus $(3 \%)$, and lastly dyspnea $(1 \%)$. A total of $34(29 \%)$ CT scans were coded as abnormal according to our previously listed criteria. Of the abnormal studies, the most common finding, present in $20(58 \%)$ cases, was isolated or multifocal periapical lucency indicative of apical periodontitis. Two (5.8\%) of the abnormal cases described multifocal apical periodontitis as well as chronic appearing osseous destruction/cortical erosion. Twelve of the abnormal cases $(35 \%)$ described findings of periapical lucency with acute appearing cortical breakthrough and resultant dental (periodontal/periapical) abscess, without trans-spatial extension or mass effect on the airway. Among 117 patients suspected of simple DA, only a single individual, a male patient, underwent a substantive management change based on facial CT results. This patient was admitted and treated in the surgical unit for trans-spatial (submandibular, sublingual, and submental) abscess, with a mild resultant mass effect on the upper airway (figure 1).

The TAT for patients undergoing facial CT had a mean value of $110( \pm 63)$ minutes and a median value of $87( \pm 71)$ minutes. Figure 2 demonstrates the distribution of TAT in a histogram display. After excluding nine patients due to insufficient radiation data, 108 observations of facial $\mathrm{CT}$ were conducted. Mean radiation dose per patient was $2.4 \mathrm{mSv}$ with a standard deviation of \pm 0.4 .

According to the Centers for Medicare and Medicaid Services (CMS) Medicare Part B Physician Fee Schedule, allowable national average facility fee for a $\mathrm{CT}$ maxillofacial without contrast is $\$ 208.85$ ( $\$ 58.03$ professional component, $\$ 150.81$ technical component); whereas, CT maxillofacial with contrast is $\$ 252.91$ (\$66.27 professional component, \$186.64 technical component). Within our cohort, 96 of 117 patients underwent contrast-enhanced CT maxillofacial, while 21 underwent non-contrast maxillofacial CT. This results in an estimated additional total cost of approximately $\$ 28,665$ within our cohort alone: $\$ 24,279$ (contrast enhanced) and $\$ 4,386$ (noncontrast). 


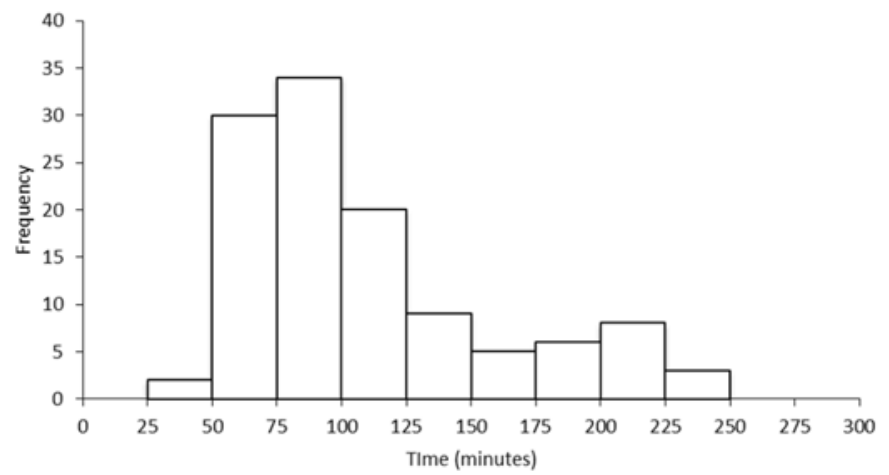

Figure 2: Turn-around time (TAT) for facial computed tomography $(\mathrm{CT})$ histogram.

\section{Discussion}

It is important to expediently identify and distinguish a simple DA from its complications. The primary reason for the distinction is that a simple DA can be conservatively managed in an outpatient setting, whereas its complications may require more extensive treatment options, and usually requires immediate life-saving interventions. The majority of simple DA is managed with surgical incision and drainage, a prescription for narcotic pain medication, an oral course of antibiotics, and an outpatient dental referral. A management change is warranted when an individual presents with an acute onset of worsening symptoms, including but not limited to a dental fistula formation and spread of infection into adjacent soft tissues spaces, leading to sublingual and submandibular abscess, cavernous sinus thrombosis, worsening cellulitis, and rarely necrotizing fasciitis.

The number of total dental ED visits in the United States has substantially increased from 1.1 million in 2000 to 2.1 million in $2010 .{ }^{1}$ In order to manage the increasing patient load, there is a growing emphasis on efficient ED patient throughput. The routine use of facial CT in patients with a suspected simple DA is an area of particular interest. The routine use of facial CT can be expensive, time consuming, and without a meaningful impact on patient management. When we further analyzed the 116 patients who had no appreciable management change based on their imaging results, the mean TAT of 110 $( \pm 63)$ minutes could have been saved. This is important, because in radiology TAT generally correlates with length of ED stay. ${ }^{1}$

Additionally, the routine use of facial CT in this population increases lifetime radiation exposure. Miglioretti et $\mathrm{al}^{6}$ reported that the ionizing radiation doses delivered by $\mathrm{CT}$ are 100 to 500 times higher than conventional radiography and are in ranges linked to an increased risk of cancer. Published literature suggests that patient history and clinical examination are sufficient to make the diagnosis of simple DA. ${ }^{7-9}$ If radiographic imaging is warranted to evaluate the severity of the abscess, then lateral and anteroposterior radiographic views, periapical, or panoramic radiography is sufficient in facilitating a diagnosis of simple DA. Implementing the aforementioned practices could have saved $99 \%$ of the patients in our investigation an average effective radiation dose of $2.4( \pm 0.4) \mathrm{mSv}$. Furthermore, when patients are discharged home with a dentist referral, standard practice in dental clinics nationwide is to generally perform additional in-office panorex examinations, without routinely reviewing prior CT images performed in the ED setting, thus exposing patients to additional radiation. ${ }^{8}$ Putting these numbers into perspective, $259.2 \mathrm{mSv}$ worth of radiation could have been spared in our study patient population.

Patients with NTDC often present to the ED as their primary source of care. ${ }^{1,10,11}$ This influx of visits is financially straining to the limited resources of the ED. Using data analyzed in our investigation; a few important financial concerns can be addressed. Based on the CMS Medicare Part B Physician Fee Schedule, the average allowable facility payments for noncontrast and contrast-enhanced CT maxillofacial are \$208.85 (\$58.03 professional component, \$150.81 technical component) and \$252.91 (\$66.27 professional component, $\$ 186.64$ technical component), respectively. ${ }^{12}$ The vast majority of patients within our cohort underwent contrastenhanced CT, as outlined in the Results section. Considering there was only one management change, approximately $\$ 28,400$ could have been spared to the paying parties in our study alone. While these costs in a multi-billion dollar healthcare industry might not seem significant, when practice patterns are evaluated on a national scale, the potential for substantial cost savings are at stake.

\section{Limitations}

This study was conducted by reviewing patient records at a single teaching hospital. The sample size for this study was modest, and all patients in the study presented to the ED. While our emergency department is a nationwide leader in several important metrics, it is important to note that practice parameters vary widely across the nation and even within this region. Furthermore, some ED settings have on-call dentistry services or Oral-Maxillofacial Surgery (OMFS) to evaluate patients with dental complaints, such as the cohort included in our study, while some do not. In our setting, on-call dentistry is not available in the ED; however, there is an OMFS service. Individual ED practitioners manage patients differently, and in some cases, consult requests to the OMFS service was placed prior to the imaging being performed; although, in most cases it was either not requested or was requested after imaging results had returned. Given this significant variability, our results may lack external validity in some settings and may not be generalizable nationwide.

Another limitation was our lack of follow-up data, as the change in management included in the study was evaluated solely based on the patient records at our hospital. Neither the patients nor the dental healthcare providers whom patients presumably saw in follow-up were contacted in regards to management changes outside the hospital. In our study, we 
did not consider whether the patients were covered under dental or health insurance. It is important to note that our academic hospital is within the heart of a major metropolitan city in a major urban center. Furthermore, the hospital in question was previously a standalone private hospital before being acquired by a larger academic network within the last 15 years. This makes the setting unique in that there is a significant high-income/insured patient base admitted to the hospital. However, given the proximity of the emergency department to a major urban/low income center, a higher than expected cohort of uninsured/indigent patients are seen in the ED. Future studies might benefit from a prospective study design and include an extensive patient follow-up plan to determine any changes in management down the road.

\section{Conclusion}

The routine use of facial $\mathrm{CT}$ in the initial evaluation of a clinically suspected simple DA is of low diagnostic yield. Furthermore, the use of facial CT as a primary investigation puts a strain on both hospital resources as well as the patient population presenting to the ED. It negatively impacts the healthcare system by contributing to an increase in TAT, exposing patients to unnecessary radiation, and risk of financial burden. It is suggested that healthcare providers opt for the use of panoramic radiographs in suspected cases, as these are considered the gold standard for the evaluation of suspected simple DA.

\section{References}

1. Wall T. Recent trends in dental emergency department visits in the United States:1997/1998 to 2007/2008. J Public Health Dent. 2012;72:216-220.

2. Shama SA. Periapical abscess of the maxillary teeth and its fistulizations: Multi-detector CT study. Alexandria Journal of Medicine. 2012;49:273-279.

3. Okunseri C, Okunseri E, Fischer MC, Sadeghi SN, Xiang Q, Szabo A. Nontraumatic dental condition-related visits to emergency departments on weekdays, weekends and night hours: findings from the National Hospital Ambulatory Medical Care survey. Clin Cosmet Investig Dent. 2013;5:6976.

4. Shuaib W, Johnson JO, Pande V, Salastekar N, Kang J, He Q, Khosa F. Ventriculoperitoneal shunt malfunction: cumulative effect of cost, radiation, and turnaround time on the patient and the health care system. AJR Am J Roentgenol. 2014;202:13-17.

5. Khan A, Khosa F, Nasir K, Yassin A, Clouse ME. Comparison of radiation dose and image quality: 320-MDCT versus 64-MDCT coronary angiography. AJR Am J Roentgenol. 2011;197:163-168.

6. Miglioretti DL, Johnson E, Williams A, Greenlee RT, Weinmann $\mathrm{S}$, Solberg LI, Feigelson HS. The use of computed tomography in pediatrics and the associated radiation exposure and estimated cancer risk. JAMA Pediatr. 2013;167:700-707.

7. Dahlén G. Microbiology and treatment of dental abscesses and periodontal-endodontic lesions. Periodontol 2000. 2002;28:206-239.

8. Krebs KA, Clem DS $3^{\text {rd }}$; American Academy of Periodontology. Guidelines for the management of patients with periodontal diseases. J Periodontol. 2006;77:1607-1611.

9. Ferrera PC, Busino LJ, Snyder HS. Uncommon complications of odontogenic infections. Am J Emerg Med. 1996;14:317-322.
10. Tang N, Stein J, Hsia RY, Maselli JH, Gonzales R. Trends and characteristics of US emergency department visits, 19972007. JAMA. 2010;304:664-670.

11. Wilber ST, Gerson LW, Terrell KM, Carpenter CR, Shah MN, Heard K, Hwang U. Geriatric emergency medicine and the 2006 Institute of Medicine reports from the Committee on the Future of Emergency Care in the U.S. health system. Acad Emerg Med. 2006;13:1345-1351.

12. Centers for Medicare and Medicaid Services. Physician Fee Schedule Search. Available at: http://www.cms.gov/apps/ physician-fee-schedule/search/search-criteria.aspx. Accessed July 9, 2014.

\section{Author Affiliations}

Waqas Shuaib, MD*; Mariyam Hashmi, MD';

Arvind Vijayasarathi, $M D, M P H, M B A^{*}$; Jay Arunkumar, DMD ; Sabeen Tiwana ${ }^{\xi}$, DMD; Faisal Khosa, $M D^{1}$

"Department of Radiology and Imaging Sciences, Emory

University Hospital, Atlanta, Georgia, USA

'Dow International Medical College, DUHS, Karachi,

Pakistan

University of Mississippi Medical Center, Jackson, Mississippi, USA

${ }^{\S}$ Midtown Dental Practice, Atlanta, Georgia, USA 